\title{
Existence of Solutions for Nonlinear Impulsive Fractional Differential Equations with $p$-Laplacian Operator
}

\author{
Ilkay Yaslan Karaca' and Fatma Tokmak ${ }^{1,2}$ \\ ${ }^{1}$ Department of Mathematics, Ege University, Bornova, 35100 Izmir, Turkey \\ ${ }^{2}$ Department of Mathematics, Gazi University, Teknikokullar, 06500 Ankara, Turkey \\ Correspondence should be addressed to Ilkay Yaslan Karaca; ilkay.karaca@ege.edu.tr
}

Received 6 February 2014; Accepted 27 February 2014; Published 26 March 2014

Academic Editor: Santanu Saha Ray

Copyright ( $) 2014$ I. Y. Karaca and F. Tokmak. This is an open access article distributed under the Creative Commons Attribution License, which permits unrestricted use, distribution, and reproduction in any medium, provided the original work is properly cited.

This paper studies the existence of solutions for a nonlinear boundary value problem of impulsive fractional differential equations with $p$-Laplacian operator. Our results are based on some standard fixed point theorems. Examples are given to show the applicability of our results.

\section{Introduction}

Fractional calculus (differentiation and integration of arbitrary order) has proved to be an important tool in the modeling of dynamical systems associated with phenomena such as fractals and chaos. In fact, this branch of calculus has found its applications in various disciplines of science and engineering such as mechanics, electricity, chemistry, biology, economics, control theory, signal and image processing, polymer rheology, regular variation in thermodynamics, biophysics, blood flow phenomena, aerodynamics, electrodynamics of complex medium, viscoelasticity and damping, control theory, wave propagation, percolation, identification, and fitting of experimental data. Fractional differential equations serve as an excellent tool for the description of hereditary properties of various materials and processes. The interest in the study of fractional-order differential equations lies in the fact that fractional-order models are found to be more accurate than the classical integer-order models; that is, there are more degrees of freedom in the fractional-order models. In consequence, the subject of fractional differential equations is gaining more and more attention. For some recent development on the topic, see [1-11] and the references therein.

Since the $p$-Laplacian operator and fractional calculus arise from many applied fields such as turbulent filtration in porous media, blood flow problems, rheology, modeling of viscoplasticity, and material science, it is worth studying the fractional $p$-Laplacian differential equations. The research of boundary value problems for $p$-Laplacian equations of fractional order has just begun in recent years; see [12-16].

The theory of impulsive differential equations is adequate mathematical models for description of evolution processes characterized by the combination of a continuous and jumps change of their state. Impulsive differential equations have become an important area of research in recent years of the needs of modern technology, engineering, economic and physics. Moreover, impulsive differential equations are richer in applications compared to the corresponding theory of ordinary differential equations. Many of the mathematical problems encountered in the study of impulsive differential equations cannot be treated with the usual techniques within the standard framework of ordinary differential equations. For the introduction of the basic theory of impulsive equations, see [17-22] and the references therein. It is worthwhile mentioning that impulsive differential equations of fractional order have not been much studied and many aspects of these equations are yet to be explored. The recent results on impulsive fractional differential equations can be found in [23-30].

The investigation on fractional $p$-Laplacian impulsive differential equations has not been appreciated well enough. 
As far as we know, there is only one paper that has considered nonlinear fractional impulsive differential equation with $p$ Laplacian operator; see [31].

Motivated by the above mentioned work on $p$-Laplacian and impulsive boundary value problems of fractional order, in this study, we consider the following problem:

$$
\begin{array}{r}
\left(\phi_{p}\left({ }^{C} D_{0^{+}}^{\alpha} u(t)\right)\right)^{\prime}=f(t, u(t)), \quad 1<\alpha \leq 2, t \in J^{\prime}, \\
\Delta u\left(t_{k}\right)=I_{k}\left(u\left(t_{k}\right)\right), \quad \Delta u^{\prime}\left(t_{k}\right)=J_{k}\left(u^{\prime}\left(t_{k}\right)\right), \\
k=1,2, \ldots, m, \\
a u(0)-b u^{\prime}(0)=0, \quad c u(1)+d u^{\prime}(1)=0,
\end{array}
$$

where ${ }^{C} D_{0^{+}}^{\alpha}$ is the Caputo fractional derivative, $\phi_{p}$ is a $p$ Laplacian operator, $\phi_{p}(s)=|s|^{p-2} s, p>1, \phi_{p}$ is invertible, and $\left(\phi_{p}\right)^{-1}=\phi_{q}$ where $(1 / p)+(1 / q)=1, f \in \mathscr{C}([0,1] \times \mathbb{R}, \mathbb{R})$, $I_{k}, J_{k} \in \mathscr{C}(\mathbb{R}, \mathbb{R}), a, b, c, d \in[0, \infty)$ with $a c+a d+b c>0$, $J=[0,1], 0=t_{0}<t_{1}<\cdots<t_{k}<\cdots<t_{m}<t_{m+1}=1$, $J^{\prime}=J \backslash\left\{t_{1}, t_{2}, \ldots, t_{m}\right\}, \Delta u\left(t_{k}\right)=u\left(t_{k}^{+}\right)-u\left(t_{k}^{-}\right)$, where $u\left(t_{k}^{+}\right)$and $u\left(t_{k}^{-}\right)$denote the right and the left limits of $u(t)$ at $t=t_{k}(k=$ $1,2, \ldots, m)$, respectively. $\Delta u^{\prime}\left(t_{k}\right)$ have a similar meaning for $u^{\prime}(t)$.

This paper is organized as follows. In Section 2, we provide some necessary definitions and preliminary lemmas which are key tools for our main results. We give and prove our main results in Section 3. Finally, in Section 4, we give some examples to demonstrate our main results.

\section{Preliminaries}

In this section, we present auxiliary lemmas which will be used later.

Let $J_{0}=\left[0, t_{1}\right], J_{1}=\left(t_{1}, t_{2}\right], \ldots, J_{m-1}=\left(t_{m-1}, t_{m}\right], J_{m}=$ $\left(t_{m}, T\right]$, and we introduce the following spaces.

$P C(J, \mathbb{R})=\left\{u: J \rightarrow \mathbb{R} \mid u \in \mathscr{C}\left(J_{k}\right), k=\right.$ $0,1, \ldots, m$ and $u\left(t_{k}^{+}\right)$exist, $\left.k=1,2, \ldots, m\right\}$ with the norm $\|u\|=\sup _{t \in J}|u(t)|$, and $P C^{1}(J, \mathbb{R})=\{u: J \rightarrow \mathbb{R} \mid$ $u \in \mathscr{C}^{1}\left(J_{k}\right), k=0,1, \ldots, m$, and $u\left(t_{k}^{+}\right), u^{\prime}\left(t_{k}^{+}\right)$exist, $k=$ $1,2, \ldots, m\}$ with the norm $\|u\|_{P C^{1}}=\max \left\{\|u\|,\left\|u^{\prime}\right\|\right\}$. Obviously, $P C(J, \mathbb{R})$ and $P C^{1}(J, \mathbb{R})$ are Banach spaces.

Definition 1. For a continuous function $f:[0, \infty) \rightarrow R$, the Caputo derivative of fractional order $\alpha$ is defined as

$$
\begin{array}{r}
{ }^{C} D_{0^{+}}^{\alpha} f(t)=\frac{1}{\Gamma(n-\alpha)} \int_{0}^{t}(t-s)^{n-\alpha-1} f^{(n)}(s) d s, \\
n=[\alpha]+1,
\end{array}
$$

where $[\alpha]$ denotes the integer part of real number $\alpha$.
Definition 2. The Riemann-Liouville fractional integral of order $\alpha$ is defined as

$$
I_{0^{+}}^{\alpha} f(t)=\frac{1}{\Gamma(\alpha)} \int_{0}^{t}(t-s)^{\alpha-1} f(s) d s, \quad \alpha>0,
$$

provided the integral exists.

Lemma 3 (see [7]). Let $\alpha>0$; then the differential equation

$$
{ }^{C} D_{0^{+}}^{\alpha} f(t)=0
$$

has solution $f(t)=c_{0}+c_{1} t+c_{2} t^{2}+\cdots+c_{n-1} t^{n-1}, c_{i} \in \mathbb{R}$, $i=0,1,2, \ldots, n-1, n=[\alpha]+1$.

Lemma 4 (see [7]). Let $\alpha>0, f \in L(0,1)$; then

$$
{ }^{C} D_{0^{+}}^{\alpha} I_{0^{+}}^{\alpha} f(t)=f(t) .
$$

Definition 5. A function $u \in P C^{1}(J, \mathbb{R})$ with its Caputo derivative of order $\alpha$ existing on $J$ is a solution of (1) if it satisfies (1).

We need the following known results to prove the existence of solutions for (1).

Theorem 6 (see [32]). Let E be a Banach space. Assume that $\Omega$ is an open bounded subset of $E$ with $\theta \in \Omega$ and let $T: \bar{\Omega} \rightarrow E$ be a completely continuous operator such that

$$
\|T u\| \leq\|u\|, \quad \forall u \in \partial \Omega
$$

\section{Then $T$ has a fixed point in $\bar{\Omega}$.}

Theorem 7 (see [32]). Let E be a Banach space. Assume that $T: E \rightarrow E$ is a completely continuous operator and the set $V=\{u \in E \mid u=\mu T u, 0<\mu<1\}$ is bounded. Then $T$ has a fixed point in $E$.

Lemma 8. For a given $y \in \mathscr{C}[0,1]$, a function $u$ is a solution of the impulsive boundary value problem of fractional order

$$
\begin{array}{r}
\left(\phi_{p}\left({ }^{C} D_{0^{+}}^{\alpha} u(t)\right)\right)^{\prime}=y(t), \quad 1<\alpha \leq 2, t \in J^{\prime}, \\
\Delta u\left(t_{k}\right)=I_{k}\left(u\left(t_{k}\right)\right), \quad \Delta u^{\prime}\left(t_{k}\right)=J_{k}\left(u^{\prime}\left(t_{k}\right)\right), \\
k=1,2, \ldots, m, \\
a u(0)-b u^{\prime}(0)=0, \quad c u(1)+d u^{\prime}(1)=0,
\end{array}
$$


if and only if $u$ is a solution of the impulsive fractional integral equation

$$
\begin{aligned}
& \iint_{0}^{t} \frac{(t-s)^{\alpha-1}}{\Gamma(\alpha)} \phi_{q}\left(\int_{0}^{s} y(r) d r\right) d s \\
& -\frac{a t+b}{a c+a d+b c} \\
& \times \int_{t_{m}}^{1}\left(\frac{c(1-s)^{\alpha-1}}{\Gamma(\alpha)}+\frac{d(1-s)^{\alpha-2}}{\Gamma(\alpha-1)}\right) \\
& \times \phi_{q}\left(\int_{0}^{s} y(r) d r\right) d s-\mathscr{A}, \\
& t \in J_{0} \text {; } \\
& \int_{t_{k}}^{t} \frac{(t-s)^{\alpha-1}}{\Gamma(\alpha)} \phi_{q}\left(\int_{0}^{s} y(r) d r\right) d s \\
& -\frac{a t+b}{a c+a d+b c} \\
& \times \int_{t_{m}}^{1}\left(\frac{c(1-s)^{\alpha-1}}{\Gamma(\alpha)}+\frac{d(1-s)^{\alpha-2}}{\Gamma(\alpha-1)}\right) \\
& \times \phi_{q}\left(\int_{0}^{s} y(r) d r\right) d s \\
& u(t)=\left\{\begin{array}{r}
+\sum_{i=1}^{k}\left[\int_{t_{i-1}}^{t_{i}} \frac{\left(t_{i}-s\right)^{\alpha-1}}{\Gamma(\alpha)}\right. \\
\quad \times \phi_{q}\left(\int_{0}^{s} y(r) d r\right) d s
\end{array}\right. \\
& \left.+I_{i}\left(u\left(t_{i}\right)\right)\right] \\
& +\sum_{i=1}^{k-1}\left(t_{k}-t_{i}\right) \\
& \times\left[\int_{t_{i-1}}^{t_{i}} \frac{\left(t_{i}-s\right)^{\alpha-2}}{\Gamma(\alpha-1)} \phi_{q}\left(\int_{0}^{s} y(r) d r\right) d s\right. \\
& \left.+J_{i}\left(u\left(t_{i}\right)\right)\right] \\
& +\sum_{i=1}^{k}\left(t-t_{k}\right) \\
& \times\left[\int_{t_{i-1}}^{t_{i}} \frac{\left(t_{i}-s\right)^{\alpha-2}}{\Gamma(\alpha-1)} \phi_{q}\left(\int_{0}^{s} y(r) d r\right) d s\right. \\
& \left.+J_{i}\left(u\left(t_{i}\right)\right)\right]-\mathscr{A} \text {, } \\
& t \in J_{k}, \quad k=1,2, \ldots, m,
\end{aligned}
$$

where

$$
\begin{gathered}
\mathscr{A}=\frac{a t+b}{a c+a d+b c} \\
\times\left\{\sum _ { i = 1 } ^ { m } \left[\int_{t_{i-1}}^{t_{i}}\left(\frac{c\left(t_{i}-s\right)^{\alpha-1}}{\Gamma(\alpha)}+\frac{d\left(t_{i}-s\right)^{\alpha-2}}{\Gamma(\alpha-1)}\right)\right.\right. \\
\quad \times \phi_{q}\left(\int_{0}^{s} y(r) d r\right) d s \\
\left.+c I_{i}\left(u\left(t_{i}\right)\right)+d J_{i}\left(u\left(t_{i}\right)\right)\right]
\end{gathered}
$$

$$
\begin{aligned}
& +c \sum_{i=1}^{m-1}\left(t_{m}-t_{i}\right) \\
& \times\left[\int_{t_{i-1}}^{t_{i}} \frac{\left(t_{i}-s\right)^{\alpha-2}}{\Gamma(\alpha-1)} \phi_{q}\left(\int_{0}^{s} y(r) d r\right) d s\right. \\
& \left.\quad+J_{i}\left(u\left(t_{i}\right)\right)\right] \\
& +c \sum_{i=1}^{m}\left(1-t_{m}\right) \\
& \times\left[\int_{t_{i-1}}^{t_{i}} \frac{\left(t_{i}-s\right)^{\alpha-2}}{\Gamma(\alpha-1)} \phi_{q}\left(\int_{0}^{s} y(r) d r\right) d s\right. \\
& \left.\left.\quad+J_{i}\left(u\left(t_{i}\right)\right)\right]\right\} .
\end{aligned}
$$

Proof. Let $u$ be a solution of (7). Then, for $t \in J_{0}$, there exist constants $c_{0}, c_{1} \in \mathbb{R}$ such that

$$
\begin{aligned}
u(t) & =I_{0^{+}}^{\alpha} \phi_{q}\left(\int_{0}^{t} y(s) d s\right)-c_{0}-c_{1} t \\
& =\frac{1}{\Gamma(\alpha)} \int_{0}^{t}(t-s)^{\alpha-1} \phi_{q}\left(\int_{0}^{s} y(r) d r\right) d s-c_{0}-c_{1} t, \\
u^{\prime}(t) & =\frac{1}{\Gamma(\alpha-1)} \int_{0}^{t}(t-s)^{\alpha-2} \phi_{q}\left(\int_{0}^{s} y(r) d r\right) d s-c_{1} .
\end{aligned}
$$

For $t \in J_{1}$, there exist constants $d_{0}, d_{1} \in \mathbb{R}$ such that

$$
\begin{gathered}
u(t)=\frac{1}{\Gamma(\alpha)} \int_{t_{1}}^{t}(t-s)^{\alpha-1} \phi_{q}\left(\int_{0}^{s} y(r) d r\right) d s \\
-d_{0}-d_{1}\left(t-t_{1}\right), \\
u^{\prime}(t)=\frac{1}{\Gamma(\alpha-1)} \int_{t_{1}}^{t}(t-s)^{\alpha-2} \phi_{q}\left(\int_{0}^{s} y(r) d r\right) d s-d_{1} .
\end{gathered}
$$

Then we have

$$
\begin{gathered}
u\left(t_{1}^{-}\right)=\frac{1}{\Gamma(\alpha)} \int_{0}^{t_{1}}(t-s)^{\alpha-1} \phi_{q}\left(\int_{0}^{s} y(r) d r\right) d s-c_{0}-c_{1} t_{1}, \\
u\left(t_{1}^{+}\right)=-d_{0}, \\
u^{\prime}\left(t_{1}^{-}\right)=\frac{1}{\Gamma(\alpha-1)} \int_{0}^{t_{1}}\left(t_{1}-s\right)^{\alpha-2} \phi_{q}\left(\int_{0}^{s} y(r) d r\right) d s-c_{1}, \\
u^{\prime}\left(t_{1}^{+}\right)=-d_{1} .
\end{gathered}
$$


In view of $\Delta u\left(t_{1}\right)=u\left(t_{1}^{+}\right)-u\left(t_{1}^{-}\right)=I_{1}\left(u\left(t_{1}\right)\right)$ and $\Delta u^{\prime}\left(t_{1}\right)=$ $u^{\prime}\left(t_{1}^{+}\right)-u^{\prime}\left(t_{1}^{-}\right)=J_{1}\left(u\left(t_{1}\right)\right)$, we have

$$
\begin{aligned}
-d_{0}= & \frac{1}{\Gamma(\alpha)} \int_{0}^{t_{1}}(t-s)^{\alpha-1} \phi_{q}\left(\int_{0}^{s} y(r) d r\right) d s \\
& -c_{0}-c_{1} t_{1}+I_{1}\left(u\left(t_{1}\right)\right), \\
-d_{1}= & \frac{1}{\Gamma(\alpha-1)} \int_{0}^{t_{1}}\left(t_{1}-s\right)^{\alpha-2} \phi_{q}\left(\int_{0}^{s} y(r) d r\right) d s \\
& -c_{1}+J_{1}\left(u\left(t_{1}\right)\right) .
\end{aligned}
$$

Consequently,

$$
\begin{aligned}
u(t)= & \frac{1}{\Gamma(\alpha)} \int_{t_{1}}^{t}(t-s)^{\alpha-1} \phi_{q}\left(\int_{0}^{s} y(r) d r\right) d s \\
& +\frac{1}{\Gamma(\alpha)} \int_{0}^{t_{1}}(t-s)^{\alpha-1} \phi_{q}\left(\int_{0}^{s} y(r) d r\right) d s \\
& +\frac{\left(t-t_{1}\right)}{\Gamma(\alpha-1)} \int_{0}^{t_{1}}\left(t_{1}-s\right)^{\alpha-2} \phi_{q}\left(\int_{0}^{s} y(r) d r\right) d s \\
& +I_{1}\left(u\left(t_{1}\right)\right)+\left(t-t_{1}\right) J_{1}\left(u\left(t_{1}\right)\right)-c_{0}-c_{1} t, \quad t \in J_{1} .
\end{aligned}
$$

By a similar process, we can get

$$
\begin{aligned}
u(t)= & \int_{t_{k}}^{t} \frac{(t-s)^{\alpha-1}}{\Gamma(\alpha)} \phi_{q}\left(\int_{0}^{s} y(r) d r\right) d s \\
& +\sum_{i=1}^{k}\left[\int_{t_{i-1}}^{t_{i}} \frac{\left(t_{i}-s\right)^{\alpha-1}}{\Gamma(\alpha)} \phi_{q}\right. \\
& \left.\times\left(\int_{0}^{s} y(r) d r\right) d s+I_{i}\left(u\left(t_{i}\right)\right)\right] \\
& +\sum_{i=1}^{k-1}\left(t_{k}-t_{i}\right)\left[\int_{t_{i-1}}^{t_{i}} \frac{\left(t_{i}-s\right)^{\alpha-2}}{\Gamma(\alpha-1)} \phi_{q}\right. \\
& \times \sum_{i=1}^{k}\left(t-t_{k}\right)\left[\int_{t_{i-1}}^{t_{i}} \frac{\left(t_{i}-s\right)^{\alpha-2}}{\Gamma(\alpha-1)} \phi_{q}\left(\int_{0}^{s} y(r) d r\right) d s\right. \\
& \left.+J_{i}\left(u\left(t_{i}\right)\right)\right]-c_{0}-c_{1} t, \\
t \in J_{k}, k=1,2, \ldots, m . & \left.\left.k\left(t_{i}\right)\right)\right]
\end{aligned}
$$

By conditions $a u(0)-b u^{\prime}(0)=0$ and $c u(1)-d u^{\prime}(1)=0$, we have

$$
\begin{aligned}
c_{0}= & \frac{b}{a c+a d+b c} \\
& \times\left\{\int_{t_{m}}^{1}\left(\frac{c(1-s)^{\alpha-1}}{\Gamma(\alpha)}+\frac{d(1-s)^{\alpha-2}}{\Gamma(\alpha-1)}\right)\right. \\
& \quad \times \phi_{q}\left(\int_{0}^{s} y(r) d r\right) d s
\end{aligned}
$$

$$
\begin{aligned}
& +\sum_{i=1}^{m}\left[\int_{t_{i-1}}^{t_{i}}\left(\frac{c\left(t_{i}-s\right)^{\alpha-1}}{\Gamma(\alpha)}+\frac{d\left(t_{i}-s\right)^{\alpha-2}}{\Gamma(\alpha-1)}\right)\right. \\
& \times \phi_{q}\left(\int_{0}^{s} y(r) d r\right) d s \\
& \left.+c I_{i}\left(u\left(t_{i}\right)\right)+d J_{i}\left(u\left(t_{i}\right)\right)\right] \\
& +c \sum_{i=1}^{m-1}\left(t_{m}-t_{i}\right) \\
& \times\left[\int_{t_{i-1}}^{t_{i}} \frac{\left(t_{i}-s\right)^{\alpha-2}}{\Gamma(\alpha-1)} \phi_{q}\left(\int_{0}^{s} y(r) d r\right) d s\right. \\
& \left.+J_{i}\left(u\left(t_{i}\right)\right)\right] \\
& +c \sum_{i=1}^{m}\left(1-t_{m}\right) \\
& \times\left[\int_{t_{i-1}}^{t_{i}} \frac{\left(t_{i}-s\right)^{\alpha-2}}{\Gamma(\alpha-1)} \phi_{q}\left(\int_{0}^{s} y(r) d r\right) d s\right. \\
& \left.\left.+J_{i}\left(u\left(t_{i}\right)\right)\right]\right\} \text {, } \\
& c_{1}=\frac{a}{a c+a d+b c} \\
& \times\left\{\int_{t_{m}}^{1}\left(\frac{c(1-s)^{\alpha-1}}{\Gamma(\alpha)}+\frac{d(1-s)^{\alpha-2}}{\Gamma(\alpha-1)}\right)\right. \\
& \times \phi_{q}\left(\int_{0}^{s} y(r) d r\right) d s \\
& +\sum_{i=1}^{m}\left[\int_{t_{i-1}}^{t_{i}}\left(\frac{c\left(t_{i}-s\right)^{\alpha-1}}{\Gamma(\alpha)}+\frac{d\left(t_{i}-s\right)^{\alpha-2}}{\Gamma(\alpha-1)}\right)\right. \\
& \times \phi_{q}\left(\int_{0}^{s} y(r) d r\right) d s \\
& \left.+c I_{i}\left(u\left(t_{i}\right)\right)+d J_{i}\left(u\left(t_{i}\right)\right)\right] \\
& +c \sum_{i=1}^{m-1}\left(t_{m}-t_{i}\right)\left[\int_{t_{i-1}}^{t_{i}} \frac{\left(t_{i}-s\right)^{\alpha-2}}{\Gamma(\alpha-1)} \phi_{q}\right. \\
& \times\left(\int_{0}^{s} y(r) d r\right) d s \\
& \left.+J_{i}\left(u\left(t_{i}\right)\right)\right] \\
& +c \sum_{i=1}^{m}\left(1-t_{m}\right) \\
& \times\left[\int_{t_{i-1}}^{t_{i}} \frac{\left(t_{i}-s\right)^{\alpha-2}}{\Gamma(\alpha-1)} \phi_{q}\right. \\
& \left.\left.\times\left(\int_{0}^{s} y(r) d r\right) d s+J_{i}\left(u\left(t_{i}\right)\right)\right]\right\} .
\end{aligned}
$$


Substituting the value of $c_{i}(i=0,1)$ in (10) and (16), we can get (8). Conversely, assume that $u$ is a solution of the impulsive fractional integral equation (8); then by a direct computation, it follows that the solution given by (8) satisfies (7). This completes the proof.

\section{Main Results}

For the sake of convenience, we set

$$
\lambda_{1}(t)=\frac{a t+b}{a c+a d+b c}, \quad \lambda_{1}^{\prime}(t)=\lambda_{2}=\frac{a}{a c+a d+b c} .
$$

Define the operator $T: P C(J, \mathbb{R}) \rightarrow P C(J, \mathbb{R})$ as

$$
\begin{aligned}
& T u(t)=\int_{t_{k}}^{t} \frac{(t-s)^{\alpha-1}}{\Gamma(\alpha)} \phi_{q}\left(\int_{0}^{s} f(r, u(r)) d r\right) d s \\
& -\lambda_{1}(t) \int_{t_{m}}^{1}\left(\frac{c(1-s)^{\alpha-1}}{\Gamma(\alpha)}+\frac{d(1-s)^{\alpha-2}}{\Gamma(\alpha-1)}\right) \phi_{q} \\
& \times\left(\int_{0}^{s} f(r, u(r)) d r\right) d s \\
& +\sum_{i=1}^{k}\left[\int_{t_{i-1}}^{t_{i}} \frac{\left(t_{i}-s\right)^{\alpha-1}}{\Gamma(\alpha)} \phi_{q}\right. \\
& \left.\times\left(\int_{0}^{s} f(r, u(r)) d r\right) d s+I_{i}\left(u\left(t_{i}\right)\right)\right] \\
& +\sum_{i=1}^{k-1}\left(t_{k}-t_{i}\right) \\
& \times\left[\int_{t_{i-1}}^{t_{i}} \frac{\left(t_{i}-s\right)^{\alpha-2}}{\Gamma(\alpha-1)} \phi_{q}\left(\int_{0}^{s} f(r, u(r)) d r\right) d s\right. \\
& \left.+J_{i}\left(u\left(t_{i}\right)\right)\right] \\
& +\sum_{i=1}^{k}\left(t-t_{k}\right) \\
& \times\left[\int_{t_{i-1}}^{t_{i}} \frac{\left(t_{i}-s\right)^{\alpha-2}}{\Gamma(\alpha-1)} \phi_{q}\left(\int_{0}^{s} f(r, u(r)) d r\right) d s\right. \\
& \left.+J_{i}\left(u\left(t_{i}\right)\right)\right] \\
& -\lambda_{1}(t)\left\{\sum _ { i = 1 } ^ { m } \left[\int_{t_{i-1}}^{t_{i}}\left(\frac{c\left(t_{i}-s\right)^{\alpha-1}}{\Gamma(\alpha)}+\frac{d\left(t_{i}-s\right)^{\alpha-2}}{\Gamma(\alpha-1)}\right)\right.\right. \\
& \times \phi_{q}\left(\int_{0}^{s} f(r, u(r)) d r\right) d s \\
& \left.+c I_{i}\left(u\left(t_{i}\right)\right)+d J_{i}\left(u\left(t_{i}\right)\right)\right]
\end{aligned}
$$

$$
\begin{gathered}
+c \sum_{i=1}^{m-1}\left(t_{m}-t_{i}\right) \\
\times\left[\int_{t_{i-1}}^{t_{i}} \frac{\left(t_{i}-s\right)^{\alpha-2}}{\Gamma(\alpha-1)} \phi_{q}\right. \\
\times\left(\int_{0}^{s} f(r, u(r)) d r\right) d s \\
+c \sum_{i=1}^{m}\left(1-t_{m}\right) \\
\times\left[\int_{t_{i-1}}^{t_{i}} \frac{\left(t_{i}-s\right)^{\alpha-2}}{\Gamma(\alpha-1)} \phi_{q}\right. \\
\times\left(\int_{0}^{s} f(r, u(r)) d r\right) d s \\
\left.\left.+J_{i}\left(u\left(t_{i}\right)\right)\right]\right\} .
\end{gathered}
$$

Then the problem (1) has a solution if and only if the operator $T$ has a fixed point.

Lemma 9. The operator $T: P C(J, \mathbb{R}) \rightarrow P C(J, \mathbb{R})$ defined by (19) is completely continuous.

Proof. It is obvious that $T$ is continuous in view of the continuity of $f, I_{k}$, and $J_{k}$. Let $\Omega \subset P C(J, \mathbb{R})$ be bounded. Then, there exist positive constants $M_{i}>0(i=1,2,3)$ such that $|f(t, u)| \leq \phi_{p}\left(M_{1}\right),\left|I_{k}(u)\right| \leq M_{2}$ and $\left|J_{k}(u)\right| \leq M_{3}$, $\forall u \in \Omega$. Thus, $\forall u \in \Omega$, and we have

$$
\begin{aligned}
&|T u(t)| \leq \int_{t_{k}}^{t} \frac{(t-s)^{\alpha-1}}{\Gamma(\alpha)} \phi_{q}\left(\int_{0}^{s}|f(r, u(r))| d r\right) d s \\
&+\lambda_{1}(t) \int_{t_{m}}^{1}\left(\frac{c(1-s)^{\alpha-1}}{\Gamma(\alpha)}+\frac{d(1-s)^{\alpha-2}}{\Gamma(\alpha-1)}\right) \\
& \times \phi_{q}\left(\int_{0}^{s}|f(r, u(r))| d r\right) d s \\
&+ \sum_{i=1}^{k}\left[\int_{t_{i-1}}^{t_{i}} \frac{\left(t_{i}-s\right)^{\alpha-1}}{\Gamma(\alpha)}\right. \\
& \times \phi_{q}\left(\int_{0}^{s}|f(r, u(r))| d r\right) d s \\
&+\sum_{i=1}^{k-1}\left(t_{k}-t_{i}\right) \\
& \times\left[\int_{t_{i-1}}^{t_{i}} \frac{\left(t_{i}-s\right)^{\alpha-2}}{\Gamma(\alpha-1)} \phi_{q}\left(\int_{0}^{s}|f(r, u(r))| d r\right) d s\right. \\
&\left.+\left|J_{i}\left(u\left(t_{i}\right)\right)\right|\right]
\end{aligned}
$$




$$
\begin{aligned}
& +\sum_{i=1}^{k}\left(t-t_{k}\right) \\
& \times\left[\int_{t_{i-1}}^{t_{i}} \frac{\left(t_{i}-s\right)^{\alpha-2}}{\Gamma(\alpha-1)} \phi_{q}\left(\int_{0}^{s}|f(r, u(r))| d r\right) d s\right. \\
& \left.+\left|J_{i}\left(u\left(t_{i}\right)\right)\right|\right] \\
& +\lambda_{1}(t)\left\{\sum _ { i = 1 } ^ { m } \left[\int_{t_{i-1}}^{t_{i}}\left(\frac{c\left(t_{i}-s\right)^{\alpha-1}}{\Gamma(\alpha)}+\frac{d\left(t_{i}-s\right)^{\alpha-2}}{\Gamma(\alpha-1)}\right)\right.\right. \\
& \times \phi_{q}\left(\int_{0}^{s}|f(r, u(r))| d r\right) d s \\
& \left.+c\left|I_{i}\left(u\left(t_{i}\right)\right)\right|+d\left|J_{i}\left(u\left(t_{i}\right)\right)\right|\right] \\
& +c \sum_{i=1}^{m-1}\left(t_{m}-t_{i}\right) \\
& \times\left[\int_{t_{i-1}}^{t_{i}} \frac{\left(t_{i}-s\right)^{\alpha-2}}{\Gamma(\alpha-1)} \phi_{q}\right. \\
& \times\left(\int_{0}^{s}|f(r, u(r))| d r\right) d s \\
& \left.+\left|J_{i}\left(u\left(t_{i}\right)\right)\right|\right] \\
& +c \sum_{i=1}^{m}\left(1-t_{m}\right) \\
& \times\left[\int_{t_{i-1}}^{t_{i}} \frac{\left(t_{i}-s\right)^{\alpha-2}}{\Gamma(\alpha-1)} \phi_{q}\right. \\
& \times\left(\int_{0}^{s}|f(r, u(r))| d r\right) d s \\
& \left.\left.+\left|J_{i}\left(u\left(t_{i}\right)\right)\right|\right]\right\} \\
& \leq M_{1} \int_{t_{k}}^{t} \frac{(t-s)^{\alpha-1}}{\Gamma(\alpha)} d s \\
& +M_{1} \lambda_{1}(t) \int_{t_{m}}^{1}\left(\frac{c(1-s)^{\alpha-1}}{\Gamma(\alpha)}+\frac{d(1-s)^{\alpha-2}}{\Gamma(\alpha-1)}\right) d s \\
& +\sum_{i=1}^{m}\left[M_{1} \int_{t_{i-1}}^{t_{i}} \frac{\left(t_{i}-s\right)^{\alpha-1}}{\Gamma(\alpha)} d s+M_{2}\right] \\
& +\sum_{i=1}^{m-1}\left(t_{m}-t_{i}\right)\left[M_{1} \int_{t_{i-1}}^{t_{i}} \frac{\left(t_{i}-s\right)^{\alpha-2}}{\Gamma(\alpha-1)} d s+M_{3}\right] \\
& +\sum_{i=1}^{k}\left(t-t_{k}\right)\left[M_{1} \int_{t_{i-1}}^{t_{i}} \frac{\left(t_{i}-s\right)^{\alpha-2}}{\Gamma(\alpha-1)} d s+M_{3}\right]
\end{aligned}
$$$$
\begin{array}{r}
+\lambda_{1}(t)\left\{\sum _ { i = 1 } ^ { m } \left[M _ { 1 } \int _ { t _ { i - 1 } } ^ { t _ { i } } \left(\frac{c\left(t_{i}-s\right)^{\alpha-1}}{\Gamma(\alpha)}\right.\right.\right. \\
\left.+\frac{d\left(t_{i}-s\right)^{\alpha-2}}{\Gamma(\alpha-1)}\right) d s
\end{array}
$$$$
\left.+c M_{2}+d M_{3}\right]
$$$$
+c \sum_{i=1}^{m-1}\left(t_{m}-t_{i}\right)
$$$$
\times\left[M_{1} \int_{t_{i-1}}^{t_{i}} \frac{\left(t_{i}-s\right)^{\alpha-2}}{\Gamma(\alpha-1)} d s+M_{3}\right]
$$$$
+c \sum_{i=1}^{m}\left(1-t_{m}\right)
$$$$
\left.\times\left[M_{1} \int_{t_{i-1}}^{t_{i}} \frac{\left(t_{i}-s\right)^{\alpha-2}}{\Gamma(\alpha-1)} d s+M_{3}\right]\right\}
$$$$
\leq \frac{(1+m)\left(1+c \lambda_{1}(1)\right) M_{1}}{\Gamma(\alpha+1)}
$$$$
+\frac{\left[(2 m-1)\left(1+c \lambda_{1}(1)\right)+d \lambda_{1}(1)(1+m)\right] M_{1}}{\Gamma(\alpha)}
$$$$
+\left(1+c \lambda_{1}(1)\right) m M_{2}
$$$$
+\left[(2 m-1)\left(1+c \lambda_{1}(1)\right)+m d \lambda_{1}(1)\right] M_{3} \text {. }
$$

Since $t \in[0,1]$, therefore there exists a positive constant $M$, such that $\|T u\| \leq M$, which implies that the operator $T$ is uniformly bounded.

On the other hand, for any $t \in J_{k}, 0 \leq k \leq m$, we have

$$
\begin{array}{r}
\left|(T u)^{\prime}(t)\right| \leq \int_{t_{k}}^{t} \frac{(t-s)^{\alpha-2}}{\Gamma(\alpha-1)} \phi_{q}\left(\int_{0}^{s}|f(r, u(r))| d r\right) d s \\
+\lambda_{2} \int_{t_{m}}^{1}\left(\frac{c(1-s)^{\alpha-1}}{\Gamma(\alpha)}+\frac{d(1-s)^{\alpha-2}}{\Gamma(\alpha-1)}\right) \\
\times \phi_{q}\left(\int_{0}^{s}|f(r, u(r))| d r\right) d s \\
+\sum_{i=1}^{k}\left[\int_{t_{i-1}}^{t_{i}} \frac{\left(t_{i}-s\right)^{\alpha-2}}{\Gamma(\alpha-1)}\right. \\
\times \phi_{q}\left(\int_{0}^{s}|f(r, u(r))| d r\right) d s \\
\left.+\left|J_{i}\left(u\left(t_{i}\right)\right)\right|\right]
\end{array}
$$




$$
\begin{aligned}
& +\lambda_{2}\left\{\sum _ { i = 1 } ^ { m } \left[\int_{t_{i-1}}^{t_{i}}\left(\frac{c\left(t_{i}-s\right)^{\alpha-1}}{\Gamma(\alpha)}+\frac{d\left(t_{i}-s\right)^{\alpha-2}}{\Gamma(\alpha-1)}\right)\right.\right. \\
& \times \phi_{q}\left(\int_{0}^{s}|f(r, u(r))| d r\right) d s \\
& \left.+c\left|I_{i}\left(u\left(t_{i}\right)\right)\right|+d\left|J_{i}\left(u\left(t_{i}\right)\right)\right|\right] \\
& +c \sum_{i=1}^{m-1}\left(t_{m}-t_{i}\right) \\
& \times\left[\int_{t_{i-1}}^{t_{i}} \frac{\left(t_{i}-s\right)^{\alpha-2}}{\Gamma(\alpha-1)}\right. \\
& \times \phi_{q}\left(\int_{0}^{s}|f(r, u(r))| d r\right) d s \\
& \left.+\left|J_{i}\left(u\left(t_{i}\right)\right)\right|\right] \\
& +c \sum_{i=1}^{m}\left(1-t_{m}\right) \\
& \times\left[\int_{t_{i-1}}^{t_{i}} \frac{\left(t_{i}-s\right)^{\alpha-2}}{\Gamma(\alpha-1)}\right. \\
& \times \phi_{q}\left(\int_{0}^{s}|f(r, u(r))| d r\right) d s \\
& \left.\left.+\left|J_{i}\left(u\left(t_{i}\right)\right)\right|\right]\right\} \\
& \leq M_{1} \int_{t_{k}}^{t} \frac{(t-s)^{\alpha-2}}{\Gamma(\alpha-1)} d s \\
& +M_{1} \lambda_{2} \int_{t_{m}}^{1}\left(\frac{c(1-s)^{\alpha-1}}{\Gamma(\alpha)}+\frac{d(1-s)^{\alpha-2}}{\Gamma(\alpha-1)}\right) d s \\
& +\sum_{i=1}^{m}\left[M_{1} \int_{t_{i-1}}^{t_{i}} \frac{\left(t_{i}-s\right)^{\alpha-2}}{\Gamma(\alpha-1)} d s+M_{3}\right] \\
& +\sum_{i=1}^{m-1}\left(t_{m}-t_{i}\right)\left[M_{1} \int_{t_{i-1}}^{t_{i}} \frac{\left(t_{i}-s\right)^{\alpha-2}}{\Gamma(\alpha-1)} d s+M_{3}\right] \\
& +\lambda_{2}\left\{\sum _ { i = 1 } ^ { m } \left[M _ { 1 } \int _ { t _ { i - 1 } } ^ { t _ { i } } \left(\frac{c\left(t_{i}-s\right)^{\alpha-1}}{\Gamma(\alpha)}\right.\right.\right. \\
& \left.+\frac{d\left(t_{i}-s\right)^{\alpha-2}}{\Gamma(\alpha-1)}\right) d s \\
& \left.+c M_{2}+d M_{3}\right] \\
& +c \sum_{i=1}^{m-1}\left(t_{m}-t_{i}\right) \\
& \times\left[M_{1} \int_{t_{i-1}}^{t_{i}} \frac{\left(t_{i}-s\right)^{\alpha-2}}{\Gamma(\alpha-1)} d s+M_{3}\right]
\end{aligned}
$$

Hence, for $t_{1}, t_{2} \in J_{k}, t_{1}<t_{2}, 0 \leq k \leq m$, we have

$$
\left|(T u)\left(t_{2}\right)-(T u)\left(t_{1}\right)\right| \leq \int_{t_{1}}^{t_{2}}\left|(T u)^{\prime}(s)\right| d s \leq \bar{M}\left(t_{2}-t_{1}\right),
$$

which implies that $T$ is equicontinuous on all $J_{k}, k=$ $0,1,2, \ldots, m$. Thus, by the Arzela-Ascoli theorem, the operator $T: P C(J, \mathbb{R}) \rightarrow P C(J, \mathbb{R})$ is completely continuous.

Theorem 10. Let $\lim _{u \rightarrow 0} f(t, u) / \phi_{p}(u)=0, \lim _{u \rightarrow 0} I_{k}(u) / u=$ 0 , and $\lim _{u \rightarrow 0} J_{k}(u) / u=0$, and then the problem (1) has at least one solution.

Proof. Since $\lim _{u \rightarrow 0} f(t, u) / \phi_{p}(u)=0, \lim _{u \rightarrow 0} I_{k}(u) / u=0$, and $\lim _{u \rightarrow 0} J_{k}(u) / u=0$, therefore there exists a constant $r>0$ such that $|f(t, u)| \leq \phi_{p}\left(\delta_{1}\right)\left|\phi_{p}(u)\right|,\left|I_{k}(u)\right| \leq \delta_{2}|u|$, and $\left|J_{k}(u)\right| \leq \delta_{3}|u|$ for $0<|u|<r$, where $\delta_{i}>0(i=1,2,3)$ satisfy the inequality

$$
\begin{aligned}
& \left\{\frac{(1+m)\left(1+c \lambda_{1}(1)\right) \delta_{1}}{\Gamma(\alpha+1)}\right. \\
& +\frac{\left[(2 m-1)\left(1+c \lambda_{1}(1)\right)+d \lambda_{1}(1)(1+m)\right] \delta_{1}}{\Gamma(\alpha)} \\
& +\left(1+c \lambda_{1}(1)\right) m \delta_{2}+\left[(2 m-1)\left(1+c \lambda_{1}(1)\right)\right. \\
& \left.\left.+m d \lambda_{1}(1)\right] \delta_{3}\right\} \leq 1 .
\end{aligned}
$$

Let us set $\Omega=\{u \in P C(J, \mathbb{R}) \mid\|u\|<r\}$ and take $u \in$ $P C(J, \mathbb{R})$ such that $\|u\|=r$; that is, $u \in \partial \Omega$. Then, by the process used to obtain (20), we have

$$
\begin{aligned}
|T u(t)| \leq & \left\{\frac{(1+m)\left(1+c \lambda_{1}(1)\right) \delta_{1}}{\Gamma(\alpha+1)}\right. \\
& +\frac{\left[(2 m-1)\left(1+c \lambda_{1}(1)\right)+d \lambda_{1}(1)(1+m)\right] \delta_{1}}{\Gamma(\alpha)} \\
& +\left(1+c \lambda_{1}(1)\right) m \delta_{2}+\left[(2 m-1)\left(1+c \lambda_{1}(1)\right)\right. \\
& \left.\left.+m d \lambda_{1}(1)\right] \delta_{3}\right\}\|u\| .
\end{aligned}
$$


Thus, it follows that $\|T u\| \leq\|u\|, u \in \partial \Omega$. Therefore, by Theorem 6 , the operator $T$ has at least one fixed point, which in turn implies that the problem (1) has at least one solution $u \in \bar{\Omega}$.

Theorem 11. Assume that there exist positive constants $M_{i}(i=1,2,3)$ such that

$$
\begin{array}{r}
|f(t, u)| \leq \phi_{p}\left(M_{1}\right), \quad\left|I_{k}(u)\right| \leq M_{2}, \quad\left|J_{k}(u)\right| \leq M_{3}, \\
\text { for } t \in J, u \in \mathbb{R} \text { and } k=1,2, \ldots, m .
\end{array}
$$

Then the problem (1) has at least one solution.

Proof. Let us show that the set $V=\{u \in P C(J, \mathbb{R}) \mid u=$ $\mu T u, 0<\mu<1\}$ is bounded. Let $u \in V$, and then $u=\mu T u$, $0<\mu<1$. For any $t \in J$, we have

$$
\begin{aligned}
& u(t)=\int_{t_{k}}^{t} \frac{\mu(t-s)^{\alpha-1}}{\Gamma(\alpha)} \phi_{q}\left(\int_{0}^{s} f(r, u(r)) d r\right) d s \\
& -\lambda_{1}(t) \int_{t_{m}}^{1}\left(\frac{\mu c(1-s)^{\alpha-1}}{\Gamma(\alpha)}+\frac{\mu d(1-s)^{\alpha-2}}{\Gamma(\alpha-1)}\right) \\
& \times \phi_{q}\left(\int_{0}^{s} f(r, u(r)) d r\right) d s \\
& +\sum_{i=1}^{k} \mu\left[\int_{t_{i-1}}^{t_{i}} \frac{\left(t_{i}-s\right)^{\alpha-1}}{\Gamma(\alpha)}\right. \\
& \times \phi_{q}\left(\int_{0}^{s} f(r, u(r)) d r\right) d s \\
& \left.+I_{i}\left(u\left(t_{i}\right)\right)\right] \\
& +\sum_{i=1}^{k-1} \mu\left(t_{k}-t_{i}\right) \\
& \times\left[\int_{t_{i-1}}^{t_{i}} \frac{\left(t_{i}-s\right)^{\alpha-2}}{\Gamma(\alpha-1)} \phi_{q}\left(\int_{0}^{s} f(r, u(r)) d r\right) d s\right. \\
& \left.+J_{i}\left(u\left(t_{i}\right)\right)\right] \\
& +\sum_{i=1}^{k} \mu\left(t-t_{k}\right) \\
& \times\left[\int_{t_{i-1}}^{t_{i}} \frac{\left(t_{i}-s\right)^{\alpha-2}}{\Gamma(\alpha-1)} \phi_{q}\left(\int_{0}^{s} f(r, u(r)) d r\right) d s\right. \\
& \left.+J_{i}\left(u\left(t_{i}\right)\right)\right] \\
& -\lambda_{1}(t)\left\{\sum _ { i = 1 } ^ { m } \mu \left[\int_{t_{i-1}}^{t_{i}}\left(\frac{c\left(t_{i}-s\right)^{\alpha-1}}{\Gamma(\alpha)}+\frac{d\left(t_{i}-s\right)^{\alpha-2}}{\Gamma(\alpha-1)}\right)\right.\right. \\
& \times \phi_{q}\left(\int_{0}^{s} f(r, u(r)) d r\right) d s \\
& \left.+c I_{i}\left(u\left(t_{i}\right)\right)+d J_{i}\left(u\left(t_{i}\right)\right)\right]
\end{aligned}
$$

$$
\begin{gathered}
+c \sum_{i=1}^{m-1} \mu\left(t_{m}-t_{i}\right) \\
\times\left[\int_{t_{i-1}}^{t_{i}} \frac{\left(t_{i}-s\right)^{\alpha-2}}{\Gamma(\alpha-1)} \phi_{q}\right. \\
\times\left(\int_{0}^{s} f(r, u(r)) d r\right) d s \\
+c \sum_{i=1}^{m} \mu\left(1-t_{m}\right) \\
\times\left[\int_{t_{i-1}}^{t_{i}} \frac{\left(t_{i}-s\right)^{\alpha-2}}{\Gamma(\alpha-1)} \phi_{q}\right. \\
\times\left(\int_{0}^{s} f(r, u(r)) d r\right) d s \\
\left.\left.+J_{i}\left(u\left(t_{i}\right)\right)\right]\right\} .
\end{gathered}
$$

Combining (25) and (26) and employing the procedure used to obtain (20), we obtain

$$
\begin{aligned}
|u(t)|= & \mu|T u(t)| \\
\leq & \int_{t_{k}}^{t} \frac{(t-s)^{\alpha-1}}{\Gamma(\alpha)} \phi_{q}\left(\int_{0}^{s}|f(r, u(r))| d r\right) d s \\
& +\lambda_{1}(t) \int_{t_{m}}^{1}\left(\frac{c(1-s)^{\alpha-1}}{\Gamma(\alpha)}+\frac{d(1-s)^{\alpha-2}}{\Gamma(\alpha-1)}\right) \\
& +\sum_{i=1}^{k}\left[\int_{t_{i-1}}^{t_{i}} \frac{\left(t_{i}-s\right)^{\alpha-1}}{\Gamma(\alpha)}|f(r, u(r))| d r\right) d s \\
& \times \phi_{q}\left(\int_{0}^{s}|f(r, u(r))| d r\right) d s \\
& \left.+\left|I_{i}\left(u\left(t_{i}\right)\right)\right|\right] \\
& +\sum_{i=1}^{k-1}\left(t_{k}-t_{i}\right) \\
& {\left[\int_{t_{i-1}}^{t_{i}} \frac{\left(t_{i}-s\right)^{\alpha-2}}{\Gamma(\alpha-1)} \phi_{q}\left(\int_{0}^{s}|f(r, u(r))| d r\right) d s\right.} \\
& \\
& \\
& \\
& \\
& \\
& \\
& \\
& \\
& \\
& \\
&
\end{aligned}
$$




$$
\begin{aligned}
& +\sum_{i=1}^{k}\left(t-t_{k}\right) \\
& \times\left[\int_{t_{i-1}}^{t_{i}} \frac{\left(t_{i}-s\right)^{\alpha-2}}{\Gamma(\alpha-1)} \phi_{q}\left(\int_{0}^{s}|f(r, u(r))| d r\right) d s\right. \\
& \left.+\left|J_{i}\left(u\left(t_{i}\right)\right)\right|\right] \\
& +\lambda_{1}(t)\left\{\sum _ { i = 1 } ^ { m } \left[\int_{t_{i-1}}^{t_{i}}\left(\frac{c\left(t_{i}-s\right)^{\alpha-1}}{\Gamma(\alpha)}+\frac{d\left(t_{i}-s\right)^{\alpha-2}}{\Gamma(\alpha-1)}\right)\right.\right. \\
& \times \phi_{q}\left(\int_{0}^{s}|f(r, u(r))| d r\right) d s \\
& \left.+c\left|I_{i}\left(u\left(t_{i}\right)\right)\right|+d\left|J_{i}\left(u\left(t_{i}\right)\right)\right|\right] \\
& +c \sum_{i=1}^{m-1}\left(t_{m}-t_{i}\right) \\
& \times\left[\int_{t_{i-1}}^{t_{i}} \frac{\left(t_{i}-s\right)^{\alpha-2}}{\Gamma(\alpha-1)} \phi_{q}\right. \\
& \times\left(\int_{0}^{s}|f(r, u(r))| d r\right) d s \\
& \left.+\left|J_{i}\left(u\left(t_{i}\right)\right)\right|\right] \\
& +c \sum_{i=1}^{m}\left(1-t_{m}\right) \\
& \times\left[\int_{t_{i-1}}^{t_{i}} \frac{\left(t_{i}-s\right)^{\alpha-2}}{\Gamma(\alpha-1)} \phi_{q}\right. \\
& \times\left(\int_{0}^{s}|f(r, u(r))| d r\right) d s \\
& \left.\left.+\left|J_{i}\left(u\left(t_{i}\right)\right)\right|\right]\right\} \\
& \leq\left\{\frac{(1+m)\left(1+c \lambda_{1}(1)\right) M_{1}}{\Gamma(\alpha+1)}\right. \\
& +\frac{\left[(2 m-1)\left(1+c \lambda_{1}(1)\right)+d \lambda_{1}(1)(1+m)\right] M_{1}}{\Gamma(\alpha)} \\
& +\left(1+c \lambda_{1}(1)\right) m M_{2} \\
& \left.+\left[(2 m-1)\left(1+c \lambda_{1}(1)\right)+m d \lambda_{1}(1)\right] M_{3}\right\}:=M,
\end{aligned}
$$

which implies that $\|u\| \leq M$ for any $t \in J$. So, the set $V$ is bounded. Thus, by Theorem 7 , the operator $T$ has at least one fixed point. Hence the problem (1) has at least one solution.

\section{Examples}

Example 1. For $1<\alpha \leq 2$, consider the following fractional order impulsive boundary value problem:

$$
\begin{gathered}
\left(\phi_{3}\left({ }^{C} D_{0^{+}}^{\alpha} u(t)\right)\right)^{\prime}=t \arctan ^{3} u(t)+e^{2 t} u^{5}(t), \\
0<t<1, \quad t \neq t_{1}, \quad 0<t_{1}<1, \\
\Delta u\left(t_{1}\right)=\sin \left(1-\cos u\left(t_{1}\right)\right), \\
\Delta u^{\prime}\left(t_{1}\right)=e^{u^{2}\left(t_{1}\right)}-\cos ^{2} u\left(t_{1}\right), \\
u(0)-2 u^{\prime}(0)=0, \quad 3 u(1)+u^{\prime}(1)=0 .
\end{gathered}
$$

Here $m=1, p=3, a=d=1, b=2, c=3$, $f(t, u)=t \arctan ^{3} u(t)+e^{2 t} u^{5}(t), I_{1}\left(u\left(t_{1}\right)\right)=\sin (1-\cos u)$, and $J_{1}\left(u\left(t_{1}\right)\right)=e^{u^{2}}-\cos ^{2} u$. Clearly

$$
\begin{aligned}
& \lim _{u \rightarrow 0} \frac{f(t, u)}{\phi_{p}(u)}=\lim _{u \rightarrow 0} \frac{t \arctan ^{3} u(t)+e^{2 t} u^{5}(t)}{u|u|} \\
& =\left\{\begin{array}{l}
\lim _{u \rightarrow 0^{+}} \frac{t \arctan ^{3} u(t)+e^{2 t} u^{5}(t)}{u^{2}}, \\
\lim _{u \rightarrow 0^{-}} \frac{t \arctan ^{3} u(t)+e^{2 t} u^{5}(t)}{-u^{2}},
\end{array}\right. \\
& =\left\{\begin{array}{l}
0, \\
0,
\end{array}\right. \\
& =0 \text {, }
\end{aligned}
$$

$$
\begin{aligned}
\lim _{u \rightarrow 0} \frac{I_{1}(u)}{u}= & \lim _{u \rightarrow 0} \frac{\sin (1-\cos u)}{u}=0, \\
\lim _{u \rightarrow 0} \frac{J_{1}(u)}{u} & =\lim _{u \rightarrow 0} \frac{e^{u^{2}}-\cos ^{2} u}{u} \\
& =\lim _{u \rightarrow 0} 2 u e^{u^{2}}+2 \cos u \sin u=0 .
\end{aligned}
$$

Furthermore, in this case, $\delta_{i}(i=1,2)$ given by (23) satisfy the inequality

$$
\left(\frac{19}{5 \Gamma(\alpha+1)}+\frac{5}{2 \Gamma(\alpha)}\right) \delta_{1}+\frac{19}{10} \delta_{2}+\frac{11}{5} \delta_{3} \leq 1 .
$$

Thus all the assumptions of Theorem 10 hold. Hence, the conclusion of Theorem 10 applies and the impulsive fractional boundary value problem (28) has at least one solution.

Example 2. Consider the following fractional order impulsive boundary value problem:

$$
\begin{gathered}
\left(\phi_{4}\left({ }^{C} D_{0^{+}}^{\alpha} u(t)\right)\right)^{\prime}=\frac{e^{3 t} \cos ^{3} u(t)}{1+u^{2}(t)}, \quad 0<t<1, t \neq \frac{1}{3}, \\
\Delta u\left(\frac{1}{3}\right)=\frac{2 \cos ^{2} u(1 / 3)}{u^{2}(1 / 3)+5} \\
\Delta u^{\prime(1 / 3)}=e^{-2 u^{2}(1 / 3)}\left(1+\sin \left(1+e^{2 u(1 / 3)}\right)\right) \\
2 u(0)-u^{\prime}(0)=0, \quad u(1)+5 u^{\prime}(1)=0 .
\end{gathered}
$$


Here $1<\alpha \leq 2, m=1, p=4, a=2, b=c=1$, $d=5,|f(t, u)|=\left|e^{3 t} \cos ^{3} u /\left(1+u^{2}\right)\right| \leq e^{3}=\phi_{4}(e),\left|I_{1}(u)\right|=$ $\left|2 \cos ^{2} u /\left(u^{2}+5\right)\right| \leq 2 / 5$, and $\left|J_{1}(u)\right|=\left|e^{-2 u^{2}}\left(1+\sin \left(1+e^{2 u}\right)\right)\right| \leq$ 2.

In this case, $M_{1}=e, M_{2}=2 / 5, M_{3}=2$, and the conditions of Theorem 11 can readily be verified. Thus, by the conclusion of Theorem 11, the problem (28) has at least one solution.

\section{Conflict of Interests}

The authors declare that they have no conflict of interests.

\section{Authors' Contribution}

All authors contributed equally in this paper. They read and approved the paper.

\section{Acknowledgment}

The authors would like to thank the reviewers for their valuable comments and suggestions on the paper.

\section{References}

[1] R. P. Agarwal, M. Belmekki, and M. Benchohra, "A survey on semilinear differential equations and inclusions involving Riemann-Liouville fractional derivative," Advances in Difference Equations, vol. 2009, Article ID 981728, 47 pages, 2009.

[2] B. Ahmad and J. J. Nieto, "Existence results for a coupled system of nonlinear fractional differential equations with threepoint boundary conditions," Computers and Mathematics with Applications, vol. 58, no. 9, pp. 1838-1843, 2009.

[3] K. Balachandran and J. J. Trujillo, "The nonlocal Cauchy problem for nonlinear fractional integrodifferential equations in Banach spaces," Nonlinear Analysis: Theory, Methods and Applications, vol. 72, no. 12, pp. 4587-4593, 2010.

[4] M. Belmekki, J. J. Nieto, and R. Rodríguez-López, "Existence of periodic solution for a nonlinear fractional differential equation," Boundary Value Problems, Article ID 324561, 18 pages, 2009.

[5] M. Benchohra, S. Hamani, and S. K. Ntouyas, "Boundary value problems for differential equations with fractional order and nonlocal conditions," Nonlinear Analysis: Theory, Methods and Applications, vol. 71, no. 7-8, pp. 2391-2396, 2009.

[6] M. A. Darwish and S. K. Ntouyas, "On initial and boundary value problems for fractional order mixed type functional differential inclusions," Computers and Mathematics with Applications, vol. 59, no. 3, pp. 1253-1265, 2010.

[7] A. A. Kilbas, H. M. Srivastava, and J. J. Trujillo, Theory and Applications of Fractional Differential Equations, vol. 204, Elsevier, Amsterdam, The Netherlands, 2006.

[8] V. Lakshmikantham, S. Leela, and J. Vasundhara Devi, Theory of Fractional Dynamic Systems, Cambridge Academic, Cambridge, UK, 2009.

[9] J. Sabatier, O. P. Agrawal, and J. A. T. Machado, Eds., Advances in Fractional Calculus: Theoretical Developments and Applications in Physics and Engineering, Springer, Dordrecht, Germany, 2007.

[10] G. Wang, W. Liu, and C. Ren, "Existence of solutions for multipoint nonlinear differential equations of fractional orders with integral boundary conditions," Electronic Journal of Qualitative Theory of Differential Equations, vol. 54, 10 pages, 2012.

[11] S. Zhang, "Positive solutions to singular boundary value problem for nonlinear fractional differential equation," Computers and Mathematics with Applications, vol. 59, no. 3, pp. 1300-1309, 2010.

[12] G. Chai, "Positive solutions for boundary value problem of fractional differential equation with $p$-Laplacian operator," Boundary Value Problems, vol. 2012, article 18, 20 pages, 2012.

[13] T. Chen and W. Liu, "An anti-periodic boundary value problem for the fractional differential equation with a $p$-Laplacian operator," Applied Mathematics Letters, vol. 25, no. 11, pp. 16711675, 2012.

[14] T. Chen and W. Liu, "Solvability of some boundary value problems for fractional $p$-Laplacian equation," Abstract and Applied Analysis, vol. 2013, Article ID 432509, 6 pages, 2013.

[15] X. Liu, M. Jia, and X. Xiang, "On the solvability of a fractional differential equation model involving the $p$-Laplacian operator," Computers and Mathematics with Applications, vol. 64, no. 10, pp. 3267-3272, 2012.

[16] H. Xiang, J. Wang, and Z. Liu, "Existence of concave positive solutions for boundary value problem of nonlinear fractional differential equation with $p$-Laplacian operator," International Journal of Mathematics and Mathematical Sciences, vol. 2010, Article ID 495138, 17 pages, 2010.

[17] M. Akhmet, Principles of Discontinuous Dynamical Systems, Springer, New York, NY, USA, 2010.

[18] M. Benchohra, J. Henderson, and S. Ntouyas, Impulsive Differential Equations and Inclusions, vol. 2, Hindawi, New York, NY, USA, 2006.

[19] T. Jankowski, "Positive solutions to third-order impulsive Sturm-Liouville boundary value problems with deviated arguments and one-dimensional p-Laplacian," Dynamic Systems and Applications, vol. 20, no. 4, pp. 575-586, 2011.

[20] I. Y. Karaca, "On positive solutions for fourth-order boundary value problem with impulse," Journal of Computational and Applied Mathematics, vol. 225, no. 2, pp. 356-364, 2009.

[21] O. B. Ozen, I. Y. Karaca, and F. Tokmak, "Existence results for $p$-Laplacian boundary value problems of impulsivedynamic equations on time scales," Advances in Difference Equations, vol. 2013, article 334, 14 pages, 2013.

[22] A. M. Samoilenko and N. A. Perestyuk, Impulsive Differential Equations, World Scientific, Singapore, 1995.

[23] S. Abbas and M. Benchohra, "Upper and lower solutions method for impulsive partial hyperbolic differential equations with fractional order," Nonlinear Analysis: Hybrid Systems, vol. 4, no. 3, pp. 406-413, 2010.

[24] R. P. Agarwal and B. Ahmad, "Existence of solutions for impulsive anti-periodic boundary value problems of fractional semilinear evolution equations," Dynamics of Continuous, Discrete and Impulsive Systems A, vol. 18, no. 4, pp. 457-470, 2011.

[25] B. Ahmad and S. Sivasundaram, "Existence results for nonlinear impulsive hybrid boundary value problems involving fractional differential equations," Nonlinear Analysis: Hybrid Systems, vol. 3, no. 3, pp. 251-258, 2009.

[26] B. Ahmad and S. Sivasundaram, "Existence of solutions for impulsive integral boundary value problems of fractional order," Nonlinear Analysis: Hybrid Systems, vol. 4, no. 1, pp. 134$141,2010$.

[27] G. M. Mophou, "Existence and uniqueness of mild solutions to impulsive fractional differential equations," Nonlinear Analysis: 
Theory, Methods and Applications, vol. 72, no. 3-4, pp. 1604-1615, 2010.

[28] Y. Tian and Z. Bai, "Existence results for the three-point impulsive boundary value problem involving fractional differential equations," Computers and Mathematics with Applications, vol. 59, no. 8, pp. 2601-2609, 2010.

[29] G. Wang, B. Ahmad, and L. Zhang, "Some existence results for impulsive nonlinear fractional differential equations with mixed boundary conditions," Computers and Mathematics with Applications, vol. 62, no. 3, pp. 1389-1397, 2011.

[30] G. Wang, B. Ahmad, and L. Zhang, "New existence results for nonlinear impulsive integro-differential equations of fractional order with nonlocal boundary conditions," Nonlinear Studies, vol. 20, no. 1, pp. 119-130, 2013.

[31] Z. Liu, L. Lu, and I. Szántó, "Existence of solutions for fractional impulsive differential equations with $p$-Laplacian operator," Acta Mathematica Hungarica, vol. 141, no. 3, pp. 203-219, 2013.

[32] J. X. Sun, Nonlinear Functional Analysis and Its Application, Science Press, Beijing, China, 2008. 


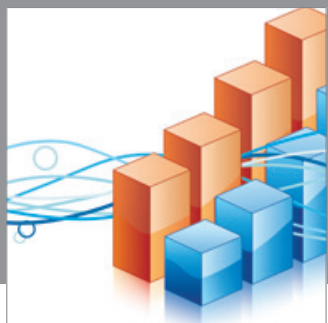

Advances in

Operations Research

mansans

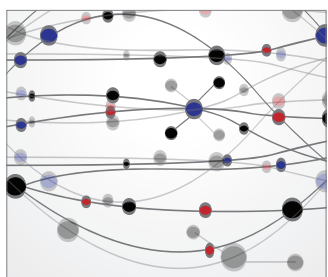

The Scientific World Journal
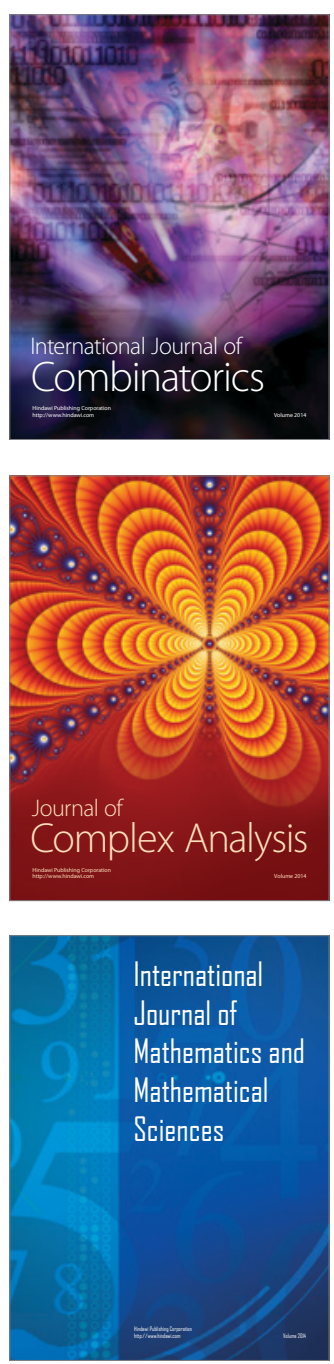
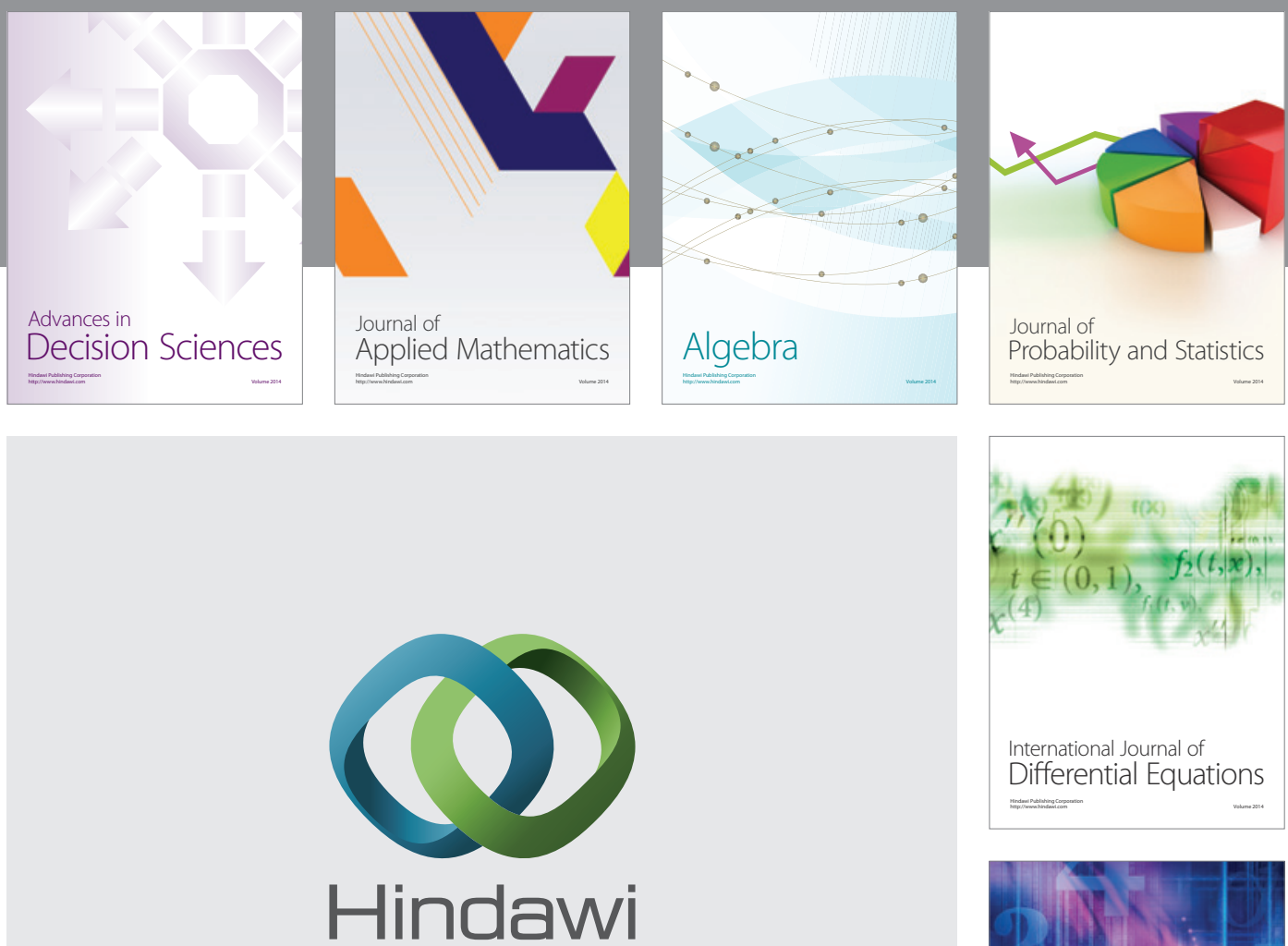

Submit your manuscripts at http://www.hindawi.com
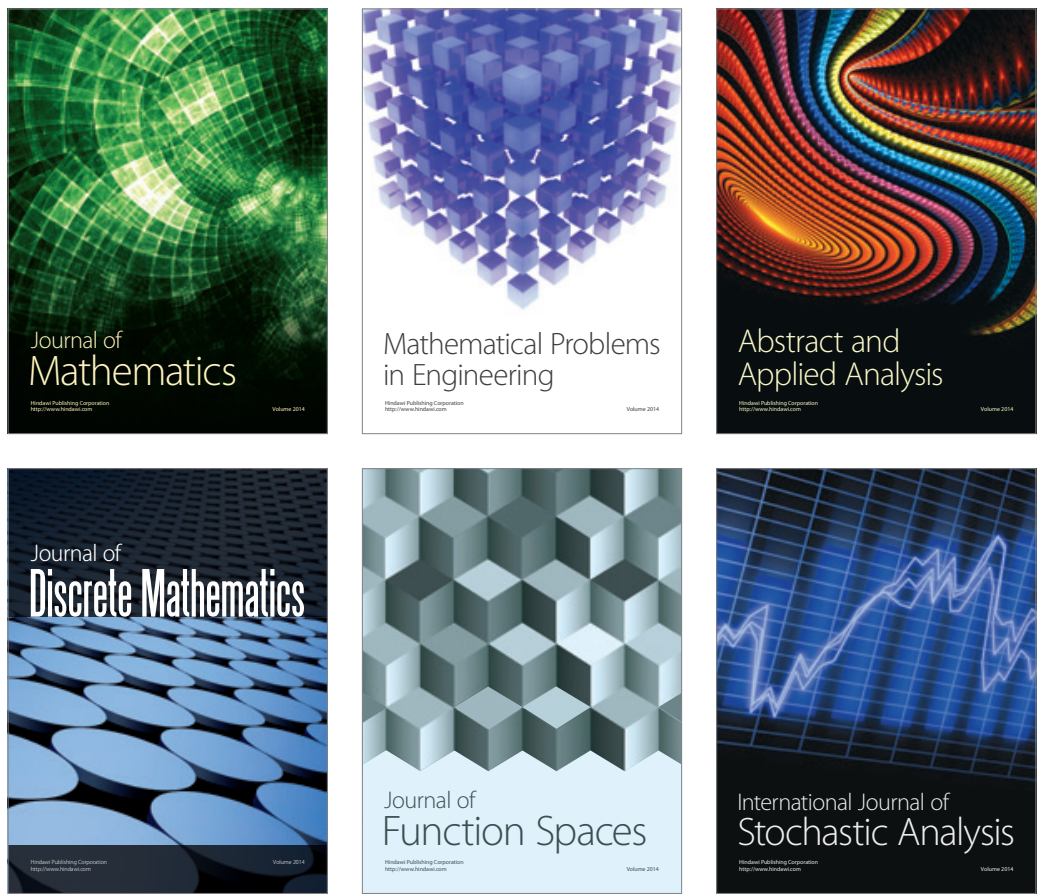

Journal of

Function Spaces

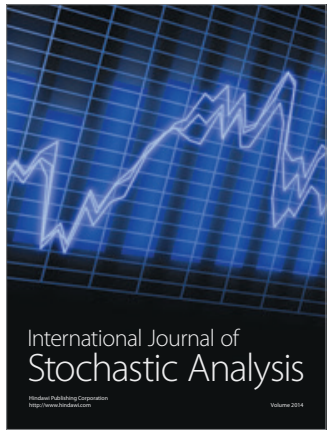

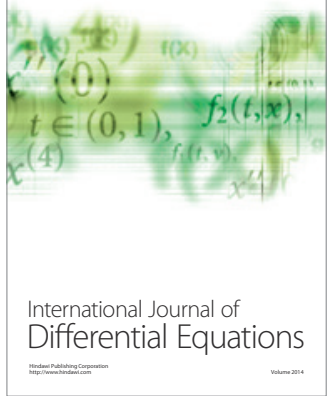
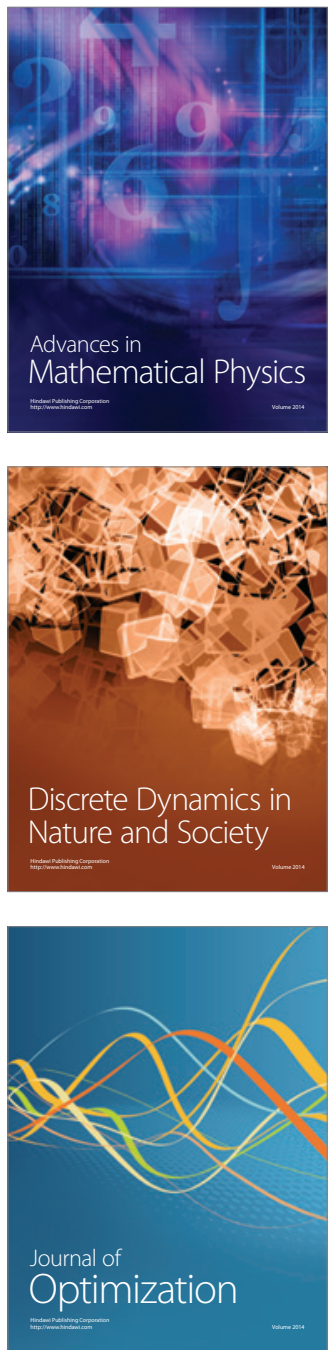